\title{
Discovery of isomers in dysprosium, holmium, and erbium isotopes with $N=94$ to 97
}

\author{
T. P. D. Swan, ${ }^{1,{ }^{*}}$ P. M. Walker, ${ }^{1,2}$ Zs. Podolyák, ${ }^{1}$ M. W. Reed, ${ }^{1}$ G. D. Dracoulis, ${ }^{3}$ G. J. Lane, ${ }^{3}$ T. Kibédi, ${ }^{3}$ and M. L. Smith ${ }^{3}$ \\ ${ }^{1}$ Department of Physics, University of Surrey, Guildford, Surrey GU2 7XH, United Kingdom \\ ${ }^{2}$ CERN, CH-1211 Geneva 23, Switzerland \\ ${ }^{3}$ Department of Nuclear Physics, RSPhysSE, Australian National University, Canberra, Australian Capital Territory 0200, Australia
}

(Received 14 December 2011; revised manuscript received 25 January 2012; published 21 February 2012)

\begin{abstract}
High-spin states in the ${ }_{68}^{164} \mathrm{Er}_{96}$ region were studied using ${ }^{9} \mathrm{Be}+{ }^{160} \mathrm{Gd}$ reactions. Pulsed beam conditions were exploited for enhanced sensitivity to delayed $\gamma$-ray transitions. New isomers were identified in ${ }^{161} \mathrm{Dy},{ }^{163} \mathrm{Ho}$, ${ }^{162} \mathrm{Er}$, and ${ }^{165} \mathrm{Er}$. The ${ }^{162} \mathrm{Er}$ isomer is interpreted to decay by $E 1$ transitions to the ground-state band with a reduced hindrance of $f_{v}=33$. A plot of $f_{v}$ values for all $\Delta K=7, E 1$ transitions from two-quasiparticle isomers to the ground-state bands of even-even nuclei reveals only a small influence from the dynamic to kinematic moment-of-inertia ratio, which provides a measure of Coriolis $K$ mixing within the ground-state band. This is interpreted to imply that the relatively low hindrances are due to $K$ mixing in the isomer, caused by a combination of Coriolis and octupole band mixing.
\end{abstract}

DOI: 10.1103/PhysRevC.85.024313 PACS number(s): 23.35.+g, 21.10.Ky, 23.20.Lv, 27.70.+q

\section{INTRODUCTION}

The slow decay rates of isomers represent an extreme of nuclear conditions. The general features of this distinctive hindered decay behavior are understood [1]. Despite these findings, interpreting the detailed differences between isomer decay rates remains a considerable challenge and provides a tantalizing opportunity to reveal less apparent features of nuclear structure.

An important degree of freedom is described by the $K$ quantum number, the projection of angular momentum on the symmetry axis of the deformed nuclear shape. Decays from high- $K$ states may be isomeric if available daughter levels represent a large change in $K$. Such decays should require a comparatively large multipole transition, $\lambda$. Nevertheless, low-multipole forbidden transitions are observed, which are hindered by the degree of forbiddenness, $v=\Delta K-\lambda$. In quantifying the hindrance of the decay, the half-life is measured relative to its Weisskopf estimate, $F_{W}=T_{1 / 2}^{\gamma} / T_{1 / 2}^{W}$. The influence of the forbiddenness on the hindered decay is described by the reduced hindrance, $f_{v}=F_{W}^{1 / v}$ [2]. A wide range of $f_{v}$ values have been measured, demanding the consideration of other variables that affect decay rates, such as the reduction in $K$ conservation caused by mixing [3].

The Coriolis effect induces $K$ mixing by diverting the motion of nucleons in a rotating nuclear core. The effect reaches its greatest intensity for nucleons in high- $j$, low- $\Omega$ orbitals, where $K=\Sigma \Omega$ [4]. Similarly, the energy of an isomer relative to the yrast line can lead to small admixtures of low- $K$ states through greater level density [5]. A third form of mixing arises through axial asymmetry in the nuclear shape, leading to a reduction in $K$ conservation [6]. Despite our understanding of $K$ mixing, additional experimental data are needed to reveal the more intricate mechanisms influencing the decay.

To observe these mechanisms, it is useful to plot $f_{v}$ values as a function of variables that quantify known mixing

*t.swan@surrey.ac.uk effects [2]. A recent publication [7] exploring the decay of an isomer in ${ }^{162}$ Dy showed the relation between $f_{v}$ and the product of the valence neutron and proton numbers, $N_{p} N_{n}$. For two-quasiparticle isomers decaying by $E 2$ transitions, $N_{p} N_{n}$ is well correlated with $f_{v}$, but reveals a small additional influence from level density effects.

We now present new isomer data for other weakly populated nuclides in the same mass region. With the focus on a $K^{\pi}=7^{-}$ isomer in ${ }^{162} \mathrm{Er}, f_{v}$ is plotted as a function of the dynamic to kinematic moment-of-inertia ratio. This ratio gives a measure of Coriolis mixing in the daughter states of isomer decays to the ground-state band $[8,9]$.

\section{EXPERIMENTAL DETAILS}

In the present study, nuclei in the ${ }_{68}^{164} \mathrm{Er}_{96}$ region were populated using ${ }^{9} \mathrm{Be}+{ }^{160} \mathrm{Gd}$ complete and incomplete fusion evaporation reactions. Pulsed and chopped beams were provided by the 14UD tandem accelerator at the Australian National University Heavy Ion Accelerator Facility [10] at an energy of $57 \mathrm{MeV}$. With the aim of measuring isomer properties in ${ }^{164} \operatorname{Er}$ [11], this beam energy provided the greatest cross section for ${ }^{164} \mathrm{Er}$ production through the ${ }^{160} \mathrm{Gd}\left({ }^{9} \mathrm{Be}, 5 n\right)$ reaction. A highly enriched $(>95 \%){ }^{160} \mathrm{Gd}$ target with an effective thickness of $4.36 \mathrm{mg} / \mathrm{cm}^{2}$ was placed at the center of the Compton suppressed CAESAR array [12], consisting of six High-Purity Germanium (HPGe) detectors at $\pm 48^{\circ}, \pm 97^{\circ}$, and $\pm 147^{\circ}$ in the beam plane and three larger HPGe detectors out of plane at $\pm 45^{\circ}$ and $+135^{\circ}$. The array includes two out of plane Low Energy Photon Spectrometer detectors at $-90^{\circ}$ and $-135^{\circ}$ for greater efficiency at low energies.

Chopped and bunched 1-ns beam pulses, with a 1.7- $\mu \mathrm{s}$ separation, were used to measure $\gamma$-ray events relative to the driving rf signal. These events were sorted into a "short time$\gamma$ " matrix (time vs energy). To measure lifetimes that were long compared to the $1.7-\mu \mathrm{s}$ pulsing, another measurement was performed where the beam was chopped ( $30 \mu \mathrm{s}$ on, $150 \mu \mathrm{s}$ off) and $\gamma$-ray times were measured using a clock register relative 


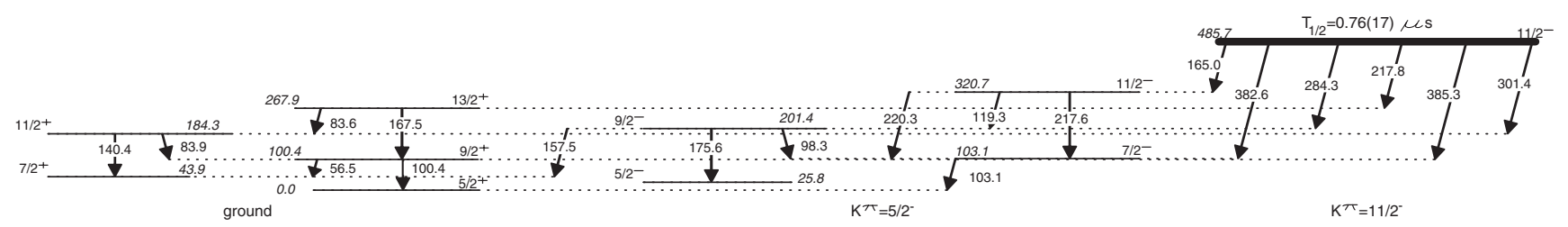

FIG. 1. Partial level scheme for ${ }_{66}^{161}$ Dy showing the newly identified isomer at $485.7 \mathrm{keV}$. All the transitions directly from the isomer are newly placed. Their relative intensities are given in Table I. Energies are in keV.

to the driving rf to create a "long time- $\gamma$ " matrix. Gamma-rays, detected between beam pulses, that were coincident within $\pm 150 \mathrm{~ns}$ were used to create an "out-of-beam $\gamma-\gamma$ " matrix. Furthermore, to detect correlated events across isomeric states, an "early-delayed $\gamma-\gamma$ " matrix was produced from events with a time difference of between 150 and $832 \mathrm{~ns}$. The usual in-beam $\gamma-\gamma$ coincidences were also studied.

\section{RESULTS}

\section{A. Isomer in ${ }^{161} \mathrm{Dy}$}

Recently, Jungclaus et al. [13] identified a rotational band above a $K^{\pi}=11 / 2^{-}$bandhead at $485.56 \mathrm{keV} \mathrm{[14]} \mathrm{in}{ }^{161} \mathrm{Dy}$. The experiment had no out-of-beam condition, and no decays out of the bandhead were observed. In the present work ${ }^{161}$ Dy is populated through the $\left({ }^{9} \mathrm{Be}, \alpha 4 n\right)$ channel. Coincidence measurements for $\gamma$-ray transitions between the low-spin members of the $K^{\pi}=5 / 2^{-}$and $K^{\pi}=5 / 2^{+}$bands (see Fig. 1) in the out-of-beam $\gamma-\gamma$ matrix identified delayed $\gamma$-ray transitions from the $485.56-\mathrm{keV}$ level, indicating an isomer. Despite a low efficiency due to a delayed coincidence width of $682 \mathrm{~ns}$, the decay branches were more clearly resolved in the "early-delayed $\gamma-\gamma$ " matrix by gating on a number of early transitions within the $K^{\pi}=11 / 2^{-}$band [13], as seen in Fig. 2 . Gamma coincidence measurements for the " $217.6+217.8$ " peak confirmed it to be a doublet.

The relative intensities of the decay branches from the isomer are shown in Table I. In agreement with Ref. [14], $\gamma$-ray

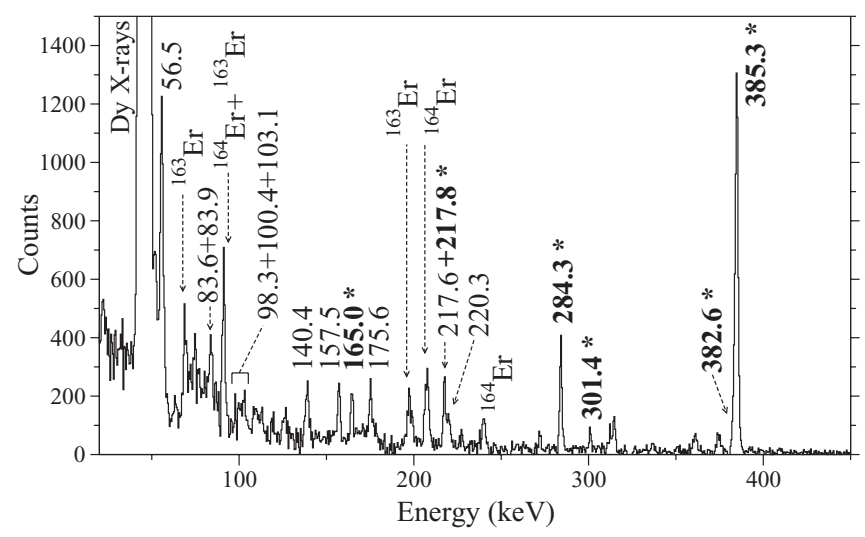

FIG. 2. Delayed coincidence spectrum for the early 156.1-, 177.3-, 198.2-, 236.2-, 375.5-, and 415.7-keV transitions, showing decays from the $K^{\pi}=11 / 2^{-}$isomer. Direct branches from the isomer are denoted by an asterisk. Random coincidences from ${ }^{164} \mathrm{Er}$ and ${ }^{163} \mathrm{Er}$ are labeled. energies place the isomeric state at 485.7(2) keV. Measurement of the half-life was hindered by the low yield and by increased contamination from other delayed components at low energies. To measure the half-life, $\gamma$-ray energy spectra were produced from contiguous time regions in the short time- and long time- $\gamma$ matrices, from which $\gamma$-ray intensity measurements were extracted for the 385.3- and $284.3-\mathrm{keV}$ branches. The half-life of the ${ }^{161}$ Dy isomer was determined (Fig. 3) to be $0.76(17) \mu \mathrm{s}$.

The $K^{\pi}=11 / 2^{-}$isomer is known to have a onequasineutron, $v 11 / 2^{-}$[505] orbital structure [14]. This structure is observed as an isomer in the nearby ${ }^{163} \mathrm{Er}$ and ${ }^{165} \mathrm{Er}$ nuclides, with half-lives of $T_{1 / 2}=0.58 \mu \mathrm{s}$ [16] and $T_{1 / 2}=$ $0.25 \mu \mathrm{s}$ [17] and excitation energies of 445 and $551 \mathrm{keV}$, respectively. In Fig. 4 transition strengths for the decays from these isomers are compared, reflecting similarity in the decay paths and probabilities and supporting the $v 11 / 2^{-}$[505] assignment for the ${ }^{161}$ Dy isomer.

\section{B. Isomers in ${ }^{163} \mathrm{Ho}$}

A $K^{\pi}=17 / 2^{+}$isomer has been observed previously in ${ }^{163} \mathrm{Ho}$ at a level energy of $1506 \mathrm{keV}$, decaying by a $973-\mathrm{keV}$ transition to the ground-state band with a half-life of $T_{1 / 2} \geqslant$ 15 ns [18] (see Fig. 5). The half-life measurement in the earlier work was limited by the loss of residual nuclei exiting the detection system. The same delayed transition was observed in the present work with an energy of $973.8 \mathrm{keV}$. A second much weaker branch from the same isomer was discovered at $581.1 \mathrm{keV}$ [see Fig. 6(a)].

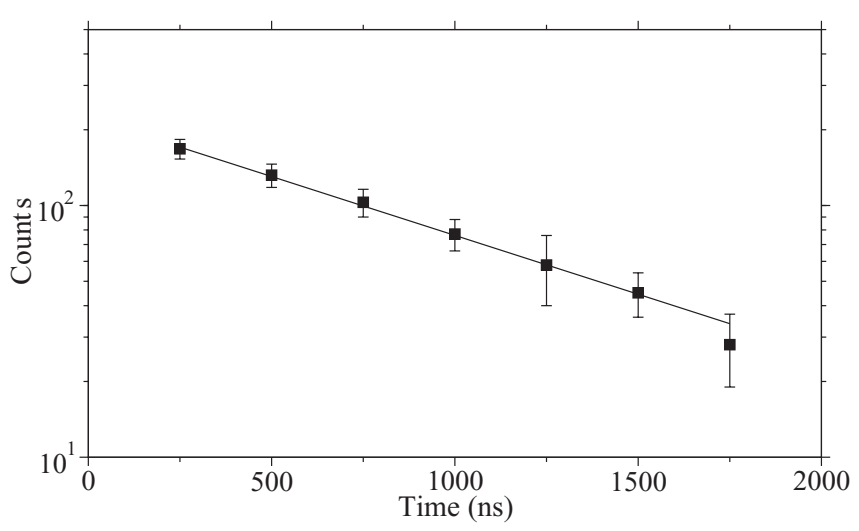

FIG. 3. One of the half-life plots for the delayed $385.3-\mathrm{keV}$ transition, using 250-ns time regions in the long time- $\gamma$ matrix. The half-life for the ${ }^{161}$ Dy isomer is $0.76(17) \mu$ s. 
TABLE I. $\gamma$-ray energy, relative $\gamma$-ray branch intensity, transition strength, and reduced hindrance measurements for direct branches from the 485.6-keV, $K^{\pi}=11 / 2^{-}$isomer in ${ }^{161} \mathrm{Dy}\left(T_{1 / 2}^{\exp }=0.76(17) \mu \mathrm{s}\right)$.

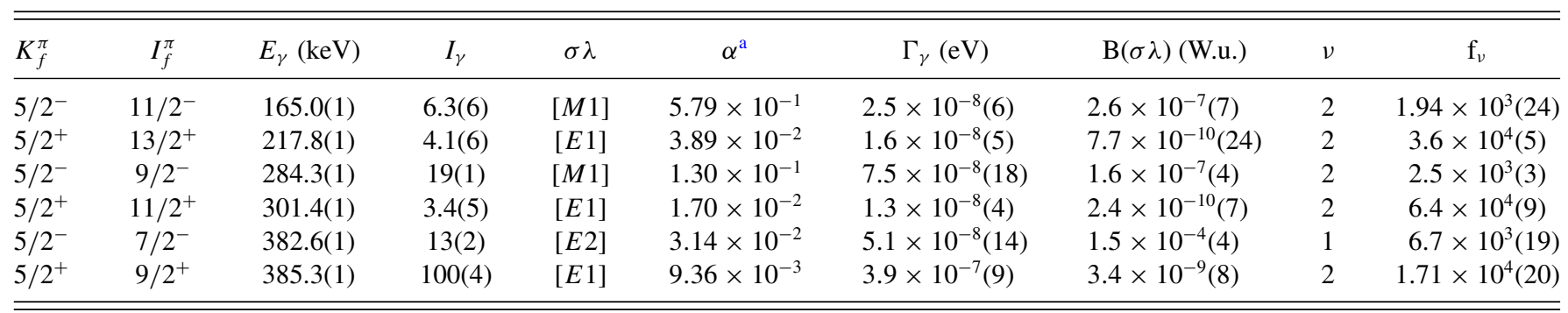

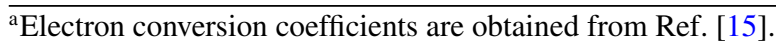

In the out-of-beam $\gamma-\gamma$ matrix, a gate on the 973.8-keV transition yielded clear evidence for a second isomer, at $2109.4(2) \mathrm{keV}$, decaying by $340.2-$ and $183.3-\mathrm{keV} \gamma$-ray transitions into the known isomer band [see Fig. 6(b)]. In Fig. 6(c), early coincidences with the delayed 340.2- and 973.8-keV transitions indicated three transitions within the 2109-keV isomer band.

The half-life of $T_{1 / 2}=0.80(15) \mu$ s for the $2109-\mathrm{keV}$ isomer was obtained from a fit to the intensity of the $973.8-\mathrm{keV}$ transition (Fig. 7), observed in $\gamma$-ray spectra produced from 156-ns-wide time regions in the short time- $\gamma$ matrix (direct branches from the isomer were heavily contaminated). Table II, shows that after $300 \mathrm{~ns}$, all of the $\gamma$-ray intensity through the $1506-\mathrm{keV}$ isomer comes from the $2109-\mathrm{keV}$ isomer, and in Fig. 6(c), the band built on the $1506-\mathrm{keV}$ isomer is not observed. This shows that the measured half-life is that of the 2109-keV isomer.

Before $300 \mathrm{~ns}$, a shorter delayed component was observed in the time spectrum for the $973.8-\mathrm{keV}$ transition, with $\gamma$-ray coincidence spectra and intensity measurements showing this to be the $1506-\mathrm{keV}$ isomer. The half-life of the $1506-\mathrm{keV}$ isomer was measured in the short time- $\gamma$ matrix with a background-subtracted gate on $973.8-\mathrm{keV} \gamma$ rays. A measure-

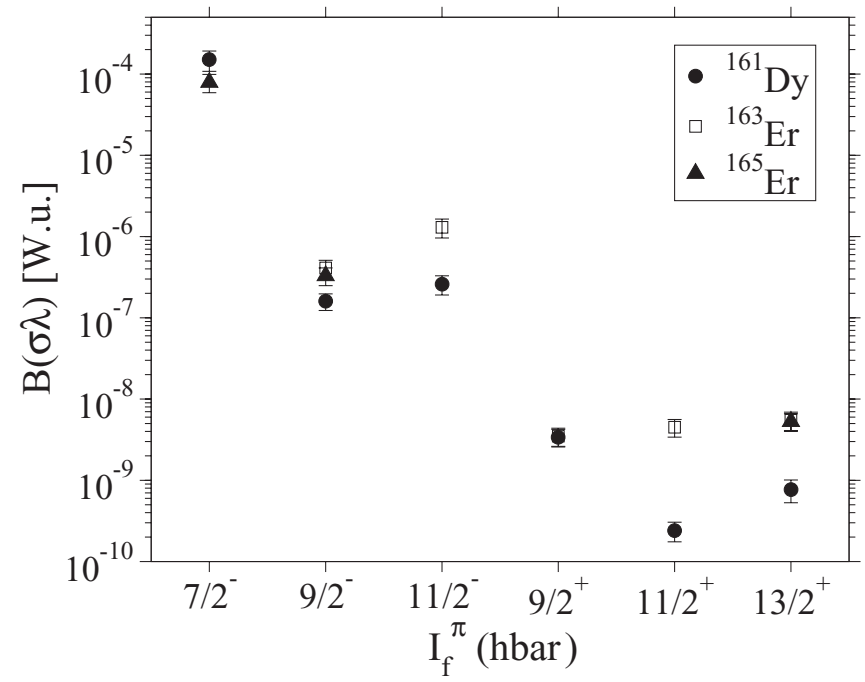

FIG. 4. Transition strengths for decay branches from the $K^{\pi}=$ $11 / 2^{-}$isomer in ${ }^{161}$ Dy compared with the corresponding decays in ${ }^{163} \operatorname{Er}[16]$ and ${ }^{165} \operatorname{Er}[17]$. ment of $T_{1 / 2}=41(11)$ ns was obtained from the fit in Fig. 8 . The fit is the sum of two exponentials, representing the two isomers, one of which is fixed at $0.80 \mu \mathrm{s}$.

Considering the intensity of the branches (Table II), the strength of the $973.8-\mathrm{keV}$ transition relative to that of the $581.1-\mathrm{keV}$ transition strongly favors a spin of $17 / 2$ for the $1506-\mathrm{keV}$ isomer, because a higher spin would require a higher multipole for the stronger transition, which is inconsistent with Weisskopf transition rate systematics [19,20]. The weakness (or absence) of the $786-\mathrm{keV}$ transition to the $17 / 2^{-}$state at $721 \mathrm{keV}$ is surprising, though a comparable situation is found for the $E 1$ decay of a $19 / 2^{-}$isomer in ${ }^{153} \mathrm{Eu} \mathrm{[21]} \mathrm{and,} \mathrm{to} \mathrm{a} \mathrm{lesser} \mathrm{extent,} \mathrm{for} \mathrm{the} 11 / 2^{-}$isomer decay in ${ }^{161}$ Dy (see Sec. III A). For the $2109-\mathrm{keV}$ isomer, the same systematics make a parent spin of $25 / 2$ unlikely, and the absence of a transition to the 19/2 level in the daughter band is consistent with such a transition having a higher multipole, favoring a spin of 23/2 for the isomer. Possible configuration assignments are discussed in Sec. IV A.

\section{Isomer in ${ }^{162} \mathrm{Er}$}

In the out-of-beam $\gamma-\gamma$ matrix, a gate on the $6^{+} \rightarrow 4^{+}$ (337.3 keV) ground-state band transition in ${ }^{162} \mathrm{Er}$ showed clear evidence for an isomer decaying by $1359.6-$ and $930.1-\mathrm{keV}$ $\gamma$-ray transitions (see Fig. 9). The half-life of the isomer

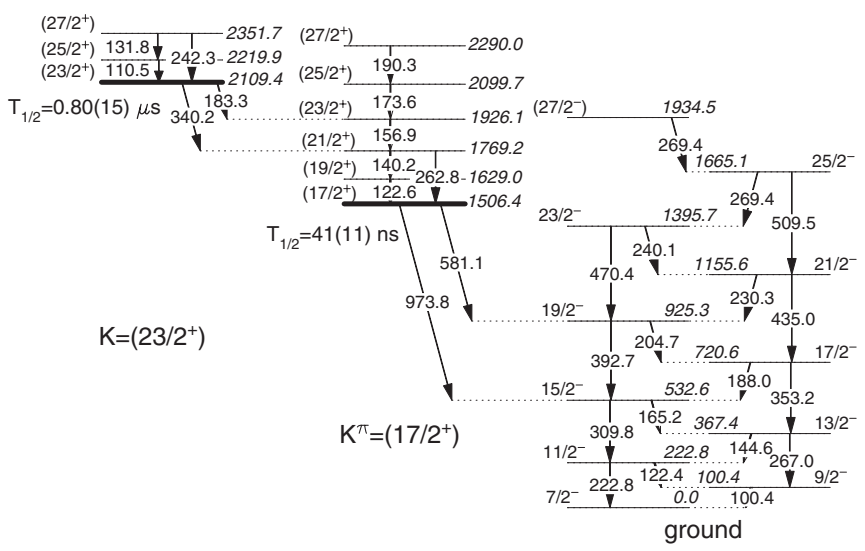

FIG. 5. Level scheme for ${ }_{67}^{163} \mathrm{Ho}$. The two half-lives are newly determined. 

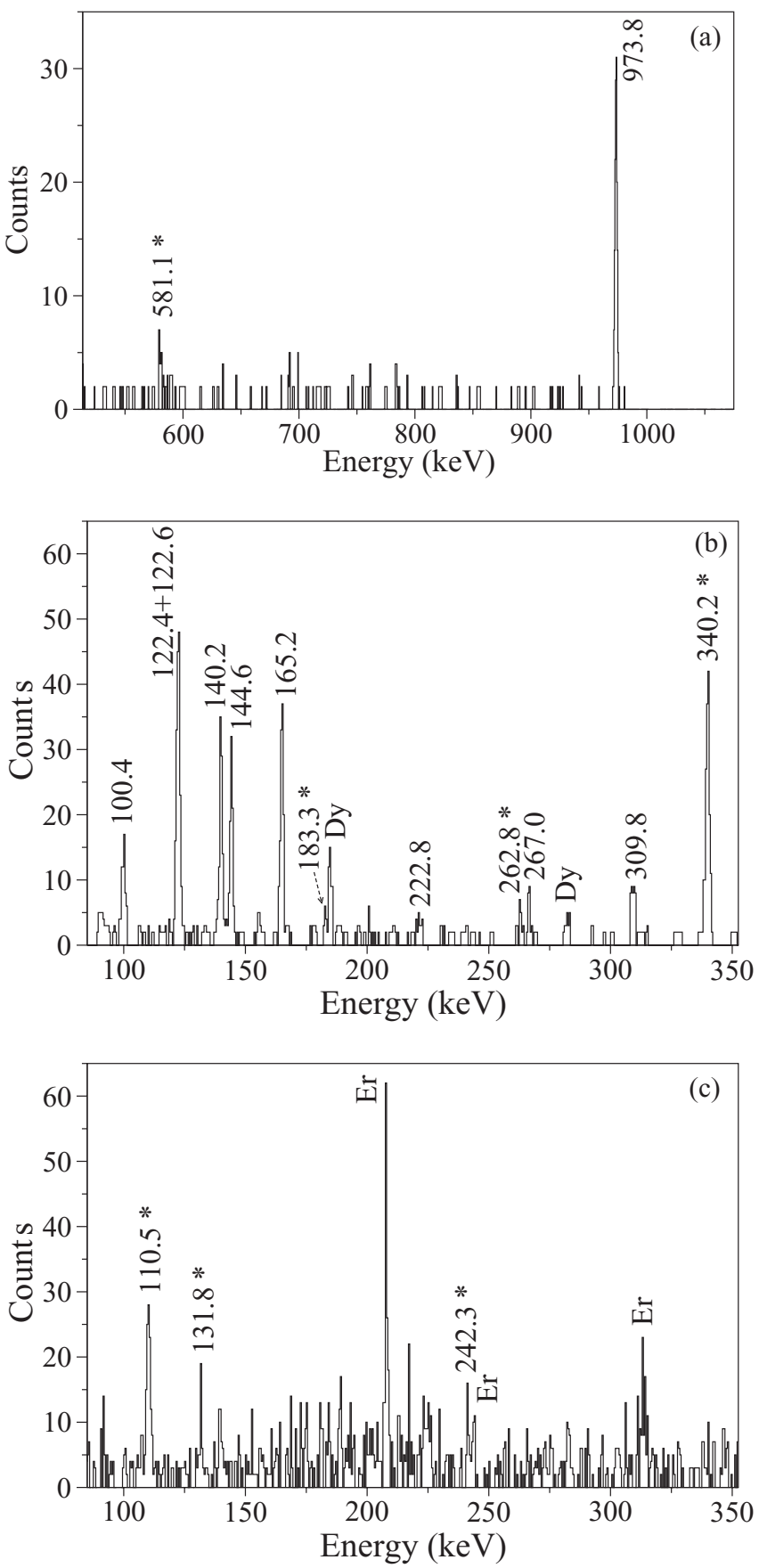

FIG. 6. (a) Coincidence spectrum for the 340.2-keV transition in the out-of-beam $\gamma-\gamma$ matrix in the range of 300-1550 ns. (b) Coincidence spectrum for the $973.8-\mathrm{keV}$ transition in the out-ofbeam $\gamma-\gamma$ matrix in the range of 300-1550 ns. (c) Early spectrum in coincidence with delayed 973.8 - or $340.2-\mathrm{keV}$ transitions. (All) New transitions are labeled with an asterisk, known ${ }^{163}$ Ho transitions are labeled with their energy, and coincidences from ${ }^{162} \mathrm{Dy}$ and ${ }^{164} \mathrm{Er}$ are labeled Dy and Er.

was measured in the short time- $\gamma$ matrix with a backgroundsubtracted gate on 1359.6-keV $\gamma$ rays. A half-life of $88(16) \mathrm{ns}$ was determined from the fit in Fig. 10.

The relative intensities, determined in the out-of-beam $\gamma-\gamma$ matrix, are shown in Table III. The $\gamma$-ray energies of these branches place the isomeric state at $2026.6(6) \mathrm{keV}$, in slight
TABLE II. Relative $\gamma$-ray intensities for transitions related to the isomers in ${ }^{163}$ Ho. Top: Delayed transitions from the two isomers, as measured in the out-of-beam $\gamma-\gamma$ matrix in the range of 300-1550 ns (intensity relative to $340.2 \mathrm{keV}$ ). Middle: Transitions above the new 2109-keV isomer (intensity relative to $110.5 \mathrm{keV}$ ). Bottom: Relative branch intensity for the newly discovered $262.8-\mathrm{keV}$ transition.

\begin{tabular}{cccccc}
\hline \hline$E_{\gamma}(\mathrm{keV})$ & $K_{i}^{\pi}$ & $K_{f}^{\pi}$ & $I_{i}^{\pi}$ & $I_{f}^{\pi}$ & $I_{\gamma}$ \\
\hline $340.2(1)$ & $\left(23 / 2^{+}\right)$ & $\left(17 / 2^{+}\right)$ & $\left(23 / 2^{+}\right)$ & $\left(21 / 2^{+}\right)$ & $100(8)$ \\
$183.3(1)$ & $\left(23 / 2^{+}\right)$ & $\left(17 / 2^{+}\right)$ & $\left(23 / 2^{+}\right)$ & $\left(23 / 2^{+}\right)$ & $6(2)$ \\
$581.1(1)$ & $\left(17 / 2^{+}\right)$ & $7 / 2^{-}$ & $\left(17 / 2^{+}\right)$ & $19 / 2^{-}$ & $12(2)$ \\
$973.8(1)$ & $\left(17 / 2^{+}\right)$ & $7 / 2^{-}$ & $\left(17 / 2^{+}\right)$ & $15 / 2^{-}$ & $89(8)$ \\
\hline $110.5(1)$ & $\left(23 / 2^{+}\right)$ & $\left(23 / 2^{+}\right)$ & $\left(25 / 2^{+}\right)$ & $\left(23 / 2^{+}\right)$ & $100(10)$ \\
$131.8(1)$ & $\left(23 / 2^{+}\right)$ & $\left(23 / 2^{+}\right)$ & $\left(27 / 2^{+}\right)$ & $\left(25 / 2^{+}\right)$ & $28(6)$ \\
$242.3(1)$ & $\left(23 / 2^{+}\right)$ & $\left(23 / 2^{+}\right)$ & $\left(27 / 2^{+}\right)$ & $\left(23 / 2^{+}\right)$ & $\geqslant 26$ \\
\hline $140.2(1)$ & $\left(17 / 2^{+}\right)$ & $\left(17 / 2^{+}\right)$ & $\left(21 / 2^{+}\right)$ & $\left(19 / 2^{+}\right)$ & $100(7)$ \\
$262.8(1)$ & $\left(17 / 2^{+}\right)$ & $\left(17 / 2^{+}\right)$ & $\left(21 / 2^{+}\right)$ & $\left(17 / 2^{+}\right)$ & $20(4)$ \\
\hline \hline
\end{tabular}

disagreement with a previous measurement of 2025.57(13) $\mathrm{keV}$ [22]. A partial level scheme is shown in Fig. 11. The two branches from the isomer have been previously reported by De Boer et al. [23] and Janssens et al. [22] with both works assigning a spin of 7 to the parent level on the basis of angular distributions. Neither work reported the level as an isomer. The level scheme is well established in these works.

Regarding the spin-parity assignment of the isomer, its level energy and decay path are similar to that of a $K^{\pi}=7^{-}$, $1985-\mathrm{keV}$ isomer in ${ }^{164} \mathrm{Er}$ with a half-life of $23 \mathrm{~ns}$ [24]. Transition strengths for the direct branches from the two isomers are compared in Fig. 12. The ${ }^{164} \mathrm{Er}$ isomer principally decays to a $K^{\pi}=5^{-}$band at the $6^{-}$and $7^{-}$levels, but equivalent branches are not observed for ${ }^{162} \mathrm{Er}$. This likely stems from the $K^{\pi}=5^{-}$band being further from yrast in ${ }^{162} \mathrm{Er}$, as expected from single-particle excitation energies for the constituent $\nu 5 / 2^{-}[523]$ and $\nu 5 / 2^{+}[642]$ nucleons, which are higher in ${ }^{161} \mathrm{Er}$ compared with ${ }^{163} \mathrm{Er}$ and ${ }^{165} \mathrm{Er}$ [25]. The transition strengths to the $K^{\pi}=0^{+}$ground-state band are similar for both isomers. We therefore favor a $K^{\pi}=7^{-}$ assignment for the ${ }^{162} \mathrm{Er}$ isomer. Its configuration and decay hindrance are discussed in Sec. IV B.

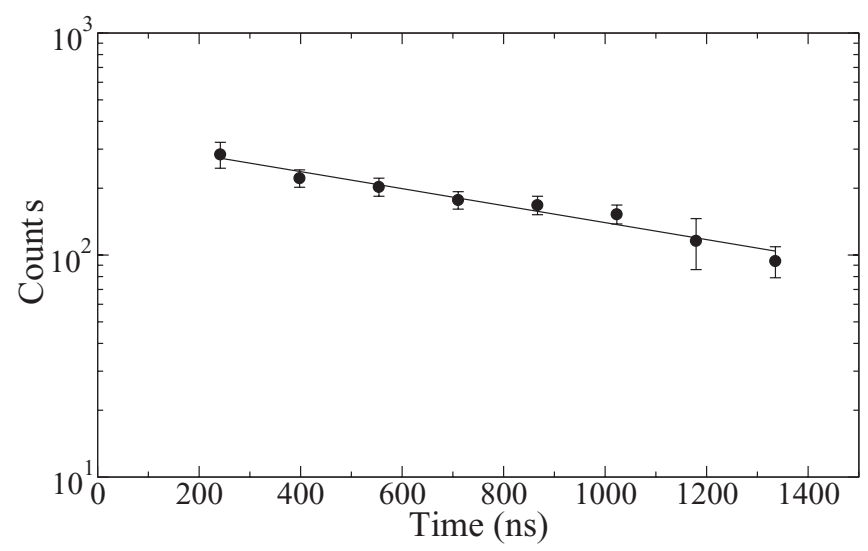

FIG. 7. Time spectrum for $973.8-\mathrm{keV} \gamma$ rays. The fitted half-life for the $2109-\mathrm{keV}$ isomer is $0.80(15) \mu \mathrm{s}$. 


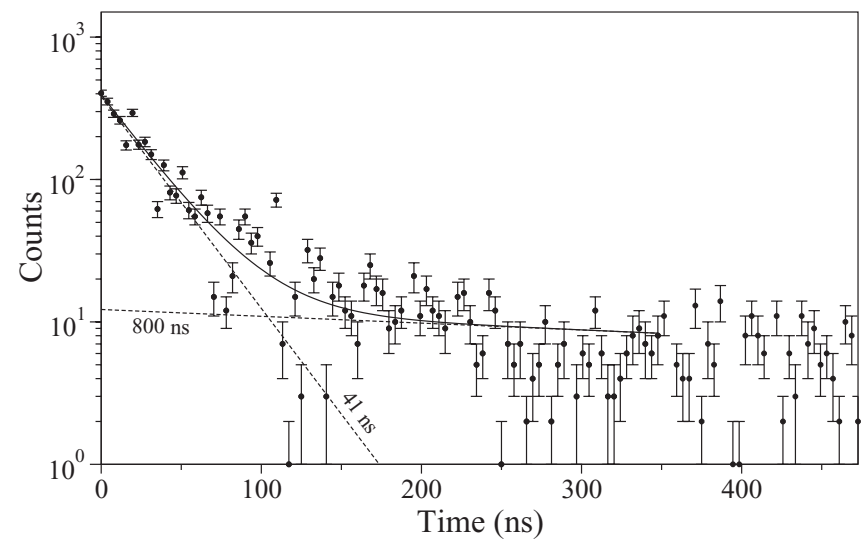

FIG. 8. Time spectrum for $973.8-\mathrm{keV} \gamma$ rays. The fitted half-life for the $1506-\mathrm{keV}$ isomer is $41(11) \mathrm{ns}$. The solid line is a fit to the data including both isomer decays.

\section{Isomer in ${ }^{165} \mathrm{Er}$}

In the out-of-beam $\gamma-\gamma$ matrix, a gate on the $13 / 2^{-} \rightarrow$ $11 / 2^{-}$transition within the known $K^{\pi}=11 / 2^{-}$isomer band in ${ }^{165} \mathrm{Er}\left(E=551 \mathrm{keV} ; T_{1 / 2}=0.25 \mu \mathrm{s}\right.$ [17]) showed evidence for another isomer, at $1823.0(2) \mathrm{keV}$, decaying by a $317.0-\mathrm{keV} \gamma$-ray transition through an unknown band into the band based on the isomer [see Fig. 13(a)]. Other direct branches from the new isomer at 1144.6 and $1050.6 \mathrm{keV}$ [see Fig. 13(b)] were assigned. In Fig. 14 gates on the delayed 317.0-, 799.8-, and 623.6-keV transitions were summed within the "early-delayed $\gamma-\gamma$ " matrix, isolating early transitions from two separate bands (bands " $\mathrm{X}$ " and "Y") that feed the isomer.

The half-life of the new isomer was measured within the short time- $\gamma$ matrix with a background-subtracted gate on the $799.8-\mathrm{keV}$ transition (the intense $317.0-\mathrm{keV}$ transition was contaminated with other delayed components). A measurement of $T_{1 / 2}=0.37(4) \mu \mathrm{s}$ was determined from the fit in Fig. 15.

A partial level scheme is shown in Fig. 16. The measured $\gamma$-ray energies place the new isomer at $1823.0(2) \mathrm{keV}$. The

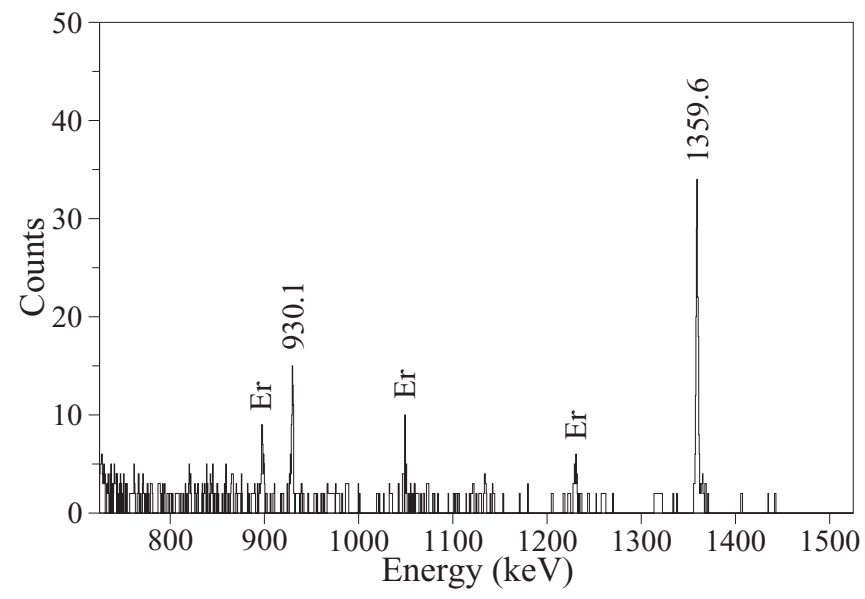

FIG. 9. Coincidence spectrum for the 337.3-keV transition in the out-of-beam $\gamma-\gamma$ matrix. Transitions in ${ }^{162} \mathrm{Er}$ are labeled with their energy. Random coincidences from ${ }^{164} \mathrm{Er}$ are labeled Er.

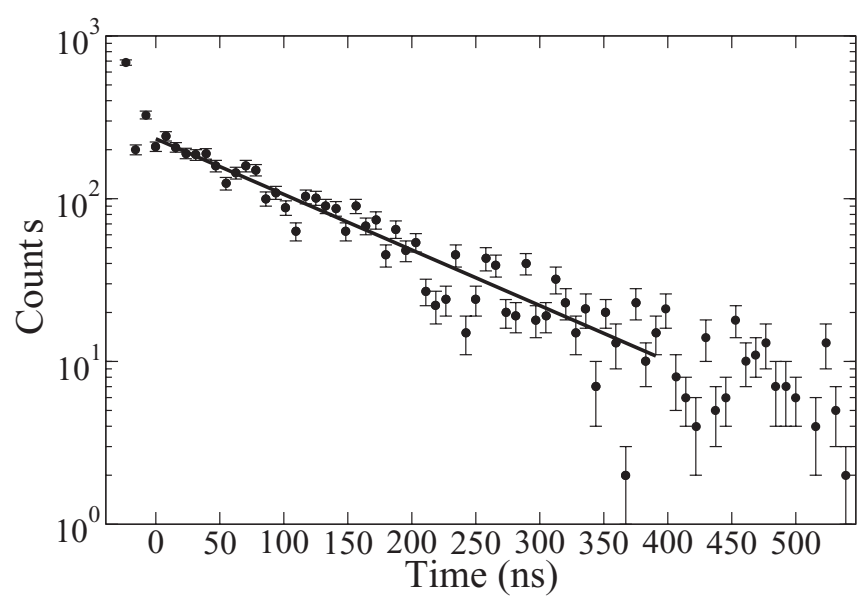

FIG. 10. Time spectrum for $1359.6-\mathrm{keV} \gamma$ rays. The fitted halflife is $88(16) \mathrm{ns}$.

1823.0(2)-keV level has been independently observed in a recent publication [26], in agreement with the excitation energy measured here, although it was not assigned as an isomer in that work. The 317.0-, 799.8-, 623.6-, 767.2-, and $188.2-\mathrm{keV}$ transitions decaying from the 1823.0(2)-keV level were also observed in that work.

The early transitions in Fig. 14 could not be detected in in-beam $\gamma-\gamma$ coincidence measurements. However, $\Delta I=2$ transitions allowed for some verification of $\Delta I=1$ placements within bands $\mathrm{X}$ and $\mathrm{Y}$. It is likely that one of the bands in early coincidence with decays from the isomer is its associated rotational band, with the other decaying into the isomeric state via an unobserved low-energy transition.

Table IV contains relative intensities for the delayed transitions. The two branches from the isomer to known-spin

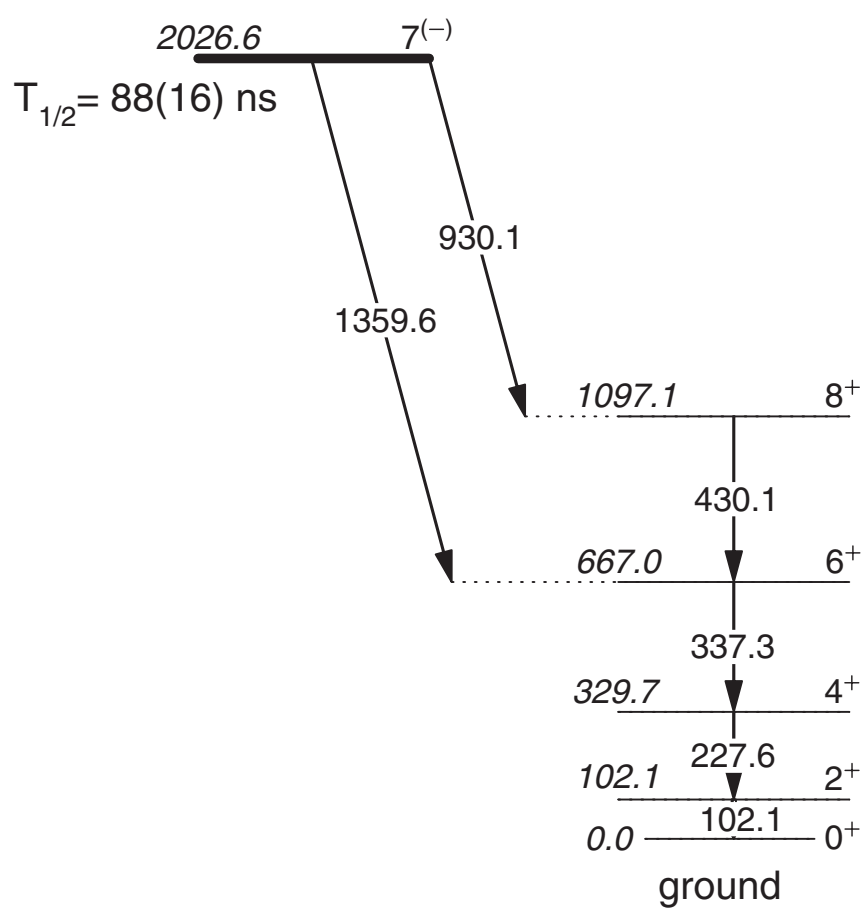

FIG. 11. Partial level scheme for ${ }^{162} \mathrm{Er}$. 


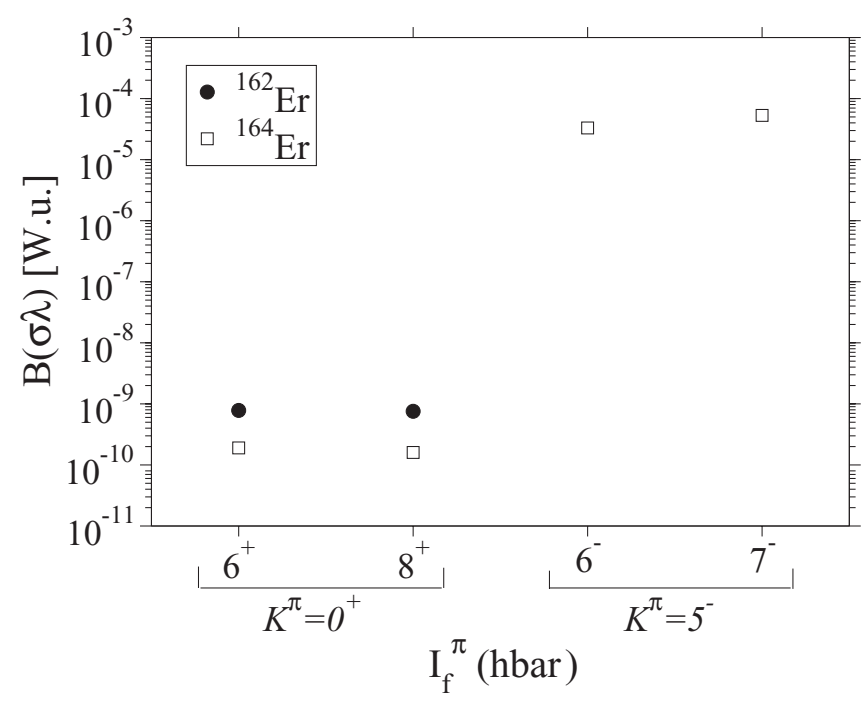

FIG. 12. Transition strengths for decay branches from the isomer in ${ }^{162} \mathrm{Er}$ compared with the decay of a $K^{\pi}=7^{-}$isomer in ${ }^{164} \mathrm{Er}$ [24] (the error bars are smaller than the data points).

levels indicate an isomer spin of between $17 / 2$ and $21 / 2$ assuming dipole or quadrupole character. Table $\mathrm{V}$ contains relative intensities for transitions within bands $\mathrm{X}$
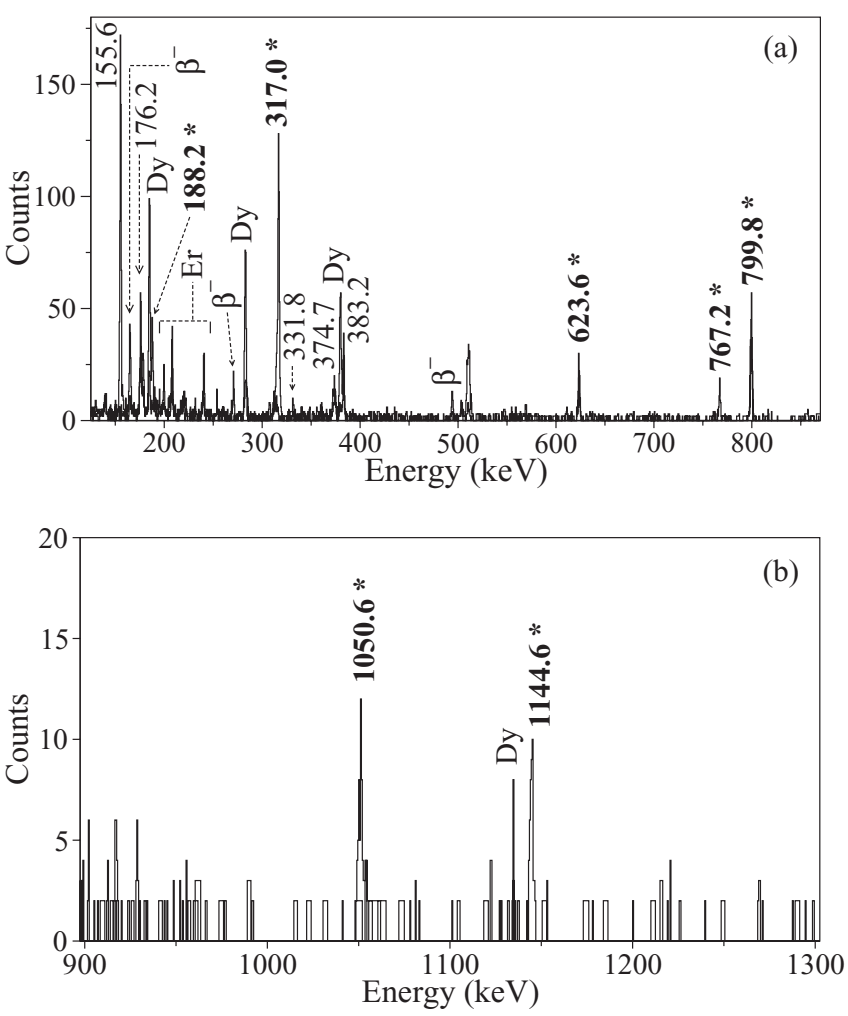

FIG. 13. Coincidence spectra in the out-of-beam $\gamma-\gamma$ matrix for transition energies of (a) 155.6 or $317.0 \mathrm{keV}$ and (b) 306.0 , $259.6,336.9$, or $400.0 \mathrm{keV}$. New transitions following the decay of the $1823.0-\mathrm{keV}$ isomer are denoted by an asterisk; known ${ }^{165} \mathrm{Er}$ transitions are labeled with their energy; and random coincidences from ${ }^{164} \mathrm{Er}$, ${ }^{162} \mathrm{Dy}$, and ${ }^{161} \mathrm{~Tb}$ are labeled $\mathrm{Er}$, Dy, and $\beta^{-}$, respectively.

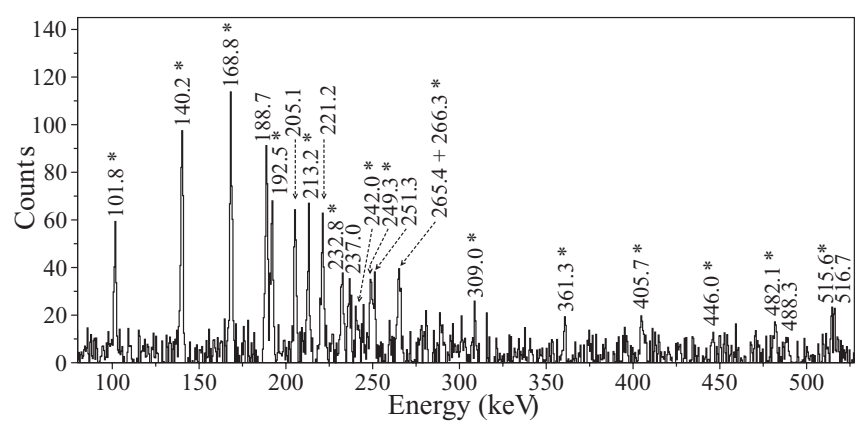

FIG. 14. Early spectrum in coincidence with delayed 317-, 800or 624-keV $\gamma$ rays. Transitions within band $\mathrm{X}$ are denoted with an asterisk. Transitions within band $\mathrm{Y}$ are also labeled.

and $\mathrm{Y}$. The tentative spin-parity assignments for bands $\mathrm{X}$ and Y (Fig. 16) are model dependent and are discussed in Sec. IV C.

\section{DISCUSSION}

The isomers with unknown structure presented in Sec. III were investigated with consideration of the intrinsic $g$ factor and alignment of the associated rotational bands, and through comparison with BCS calculations [27], which apply estimated pairing strengths to the Nilsson model to predict the energies of multi-quasiparticle states. A summary of these calculations is shown in Table VI. Residual interactions are not included in the BCS calculations, but for two-quasinucleon components the pairing energies $\left(\mathrm{G}_{n}, \mathrm{G}_{p}\right)$ are adjusted to better reproduce configurations that are favored by residual interactions. If included, the residual interaction would shift nonfavored configurations up in energy by $\sim 200 \mathrm{keV}$ [7], though in Table VI only the $7^{+}$configuration is affected in this way.

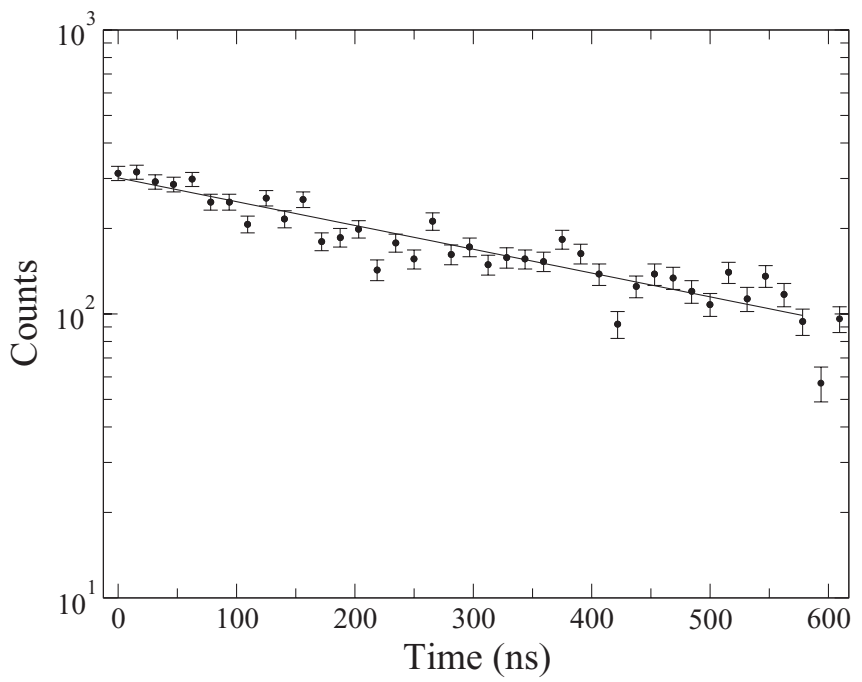

FIG. 15. Time spectrum for $799.8-\mathrm{keV} \gamma$ rays. The fitted half-life is $0.37(4) \mu \mathrm{s}$. 
TABLE III. $\gamma$-ray energy, relative $\gamma$-ray branch intensity, transition strength, and reduced hindrance measurements for direct branches from the 2026.6-keV isomer in ${ }^{162} \operatorname{Er}\left(T_{1 / 2}^{\exp }=88(16) \mathrm{ns}\right)$, based on a $K^{\pi}=7^{-}$assignment.

\begin{tabular}{|c|c|c|c|c|c|c|c|c|c|}
\hline$K_{f}^{\pi}$ & $I_{f}^{\pi}$ & $E_{\gamma}(\mathrm{keV})$ & $I_{\gamma}$ & $\sigma \lambda$ & $\alpha^{\mathrm{a}}$ & $\Gamma_{\gamma}(\mathrm{eV})$ & $\mathrm{B}(\sigma \lambda)$ (W.u.) & $v$ & $f_{v}$ \\
\hline $0^{+}$ & $8^{+}$ & $930.1(4)$ & $31(4)$ & {$[E 1]$} & $1.57 \times 10^{-3}$ & $1.2 \times 10^{-6}(3)$ & $7.7 \times 10^{-10}(18)$ & 6 & $33.1(13)$ \\
\hline $0^{+}$ & $6^{+}$ & $1359.6(2)$ & $100(7)$ & {$[E 1]$} & $8.90 \times 10^{-4}$ & $4.0 \times 10^{-6}(8)$ & $7.7 \times 10^{-10}(18)$ & 6 & $32.9(12)$ \\
\hline
\end{tabular}

aElectron conversion coefficients are obtained from Ref. [15].

\section{A. ${ }^{163} \mathrm{Ho}$}

The structural assignments of the two isomers in ${ }^{163} \mathrm{Ho}$, at 1506 and $2109 \mathrm{keV}$ (see Fig. 5), were investigated. Transition intensities favor a spin of $17 / 2$ for the $1506-\mathrm{keV}$ isomer. Indeed, Hojman et al. [18] found reasonable evidence in favor of a $17 / 2^{+}\left(\pi 7 / 2^{-}[523] \nu 5 / 2^{-}[523] \nu 5 / 2^{+}[642]\right)$ configuration based on an observation of the same structure with a similar decay in ${ }^{165} \mathrm{Tm}$ [28]. BCS calculations predict three possibilities (see Table VI) more yrast than any spin $19 / 2$ or $21 / 2$ configuration, these being the $17 / 2^{+}$candidate configuration posited by Hojman, as well as $17 / 2^{-}\left(\pi 7 / 2^{+}[404] \nu 5 / 2^{-}[523] \nu 5 / 2^{+}[642]\right)$ and $17 / 2^{-}$ $\left(\pi 7 / 2^{-}[523] \pi 7 / 2^{+}[404] \pi 3 / 2^{+}[411]\right)$, which will be referred to subsequently as $\mathrm{A}, \mathrm{B}$, and $\mathrm{C}$, respectively.

The BCS calculations predict candidate A to be closest to yrast, $440 \mathrm{keV}$ below the energy of B. As both $\mathrm{A}$ and $\mathrm{B}$ contain the $5^{-}\left(v 5 / 2^{-}[523] v 5 / 2^{+}\right.$[642]) two-quasineutron component, the energy of the isomer may be compared with the energy of that configuration in neighboring even-even nuclei. In ${ }^{162} \mathrm{Dy}$ this configuration is at an energy of $1486 \mathrm{keV}$, while for ${ }^{164} \mathrm{Er}$ it is at $1664 \mathrm{keV}$. These energies agree well with the two-quasineutron component built upon the $\pi 7 / 2^{-}[523]$ ground-state band of ${ }^{163} \mathrm{Ho}$, strongly favoring candidate A. Configuration $\mathrm{B}$ requires a much lower two-quasineutron energy of $1506-440=1066 \mathrm{keV}$, which is not favored by these systematics.

Using rotational model formulas [29], intrinsic $g$ factors were extracted from the intensities in Table II, giving $\mid g_{K}-$ $g_{R} \mid=0.22_{-0.03}^{+0.04}$, where $Q_{0}=6.4 \mathrm{~b}$ is assumed from the $\beta_{2}$ deformation calculated in Ref. [30]. We compare the experimental value with the expected intrinsic $g$ factor for the three configurations. Using intrinsic spin $g$ factors of +5.59 and -3.83 for protons and neutrons, respectively, with a quenching factor of 0.6 , and assuming an effective $g_{K}=0.08$

TABLE IV. $\gamma$-ray energy, relative $\gamma$-ray intensity, initial level energy, and final angular momentum for new delayed transitions in ${ }^{165} \mathrm{Er}$. The uncertainty in the $\gamma$-ray energies is typically $0.1 \mathrm{keV}$.

\begin{tabular}{lrcc}
\hline \hline$E_{\gamma}(\mathrm{keV})$ & \multicolumn{1}{c}{$I_{\gamma}$} & \multicolumn{1}{c}{$E_{i}$} & \multicolumn{1}{c}{$I_{f}^{\pi}$} \\
\hline 317.0 & $100(8)$ & 1823.0 & $\left(17 / 2^{-}\right)$ \\
1050.6 & $5(1)$ & 1823.0 & $17 / 2^{-}$ \\
1144.6 & $17(2)$ & 1823.0 & $19 / 2^{+}$ \\
188.2 & $13(1)$ & 1506.0 & $\left(15 / 2^{-}\right)$ \\
623.6 & $24(2)$ & 1506.0 & $15 / 2^{-}$ \\
799.8 & $62(4)$ & 1506.0 & $13 / 2^{-}$ \\
767.2 & $18(2)$ & 1317.8 & $11 / 2^{-}$ \\
\hline \hline
\end{tabular}

for $i_{13 / 2}$ neutrons [31], candidate A has $\left|g_{K}-g_{R}\right|=0.41(5)$, $\mathrm{B}$ has $\left|g_{K}-g_{R}\right|=0.13(5)$, and $\mathrm{C}$ has $\left|g_{K}-g_{R}\right|=0.84(5)$. The value of the collective $g$ factor, $g_{R}$, can vary between configurations, and, in line with Refs. [31-33], we have used $g_{R}=0.30(5)$. The $g$-factor evidence favors A and B more than $\mathrm{C}$.

Additional information about band structure comes from the degree of rotation alignment, as described in Ref. [34]. In Fig. 17, the high alignment for the $17 / 2$ isomer band suggests the presence of the $5 / 2^{+}[642]$ neutron, further ruling out configuration $\mathrm{C}$. Based on all these considerations, we tentatively favor candidate A, agreeing with the $17 / 2^{+}$ $\left(\pi 7 / 2^{-}[523] v 5 / 2^{-}[523] v 5 / 2^{+}[642]\right)$ assignment of Hojman et al. [18].

Transition intensities for the newly discovered 2109$\mathrm{keV}$ isomer indicate a bandhead spin of $23 / 2$. BCS calculations support this assignment, predicting a $23 / 2^{-}$ $\left(\pi 7 / 2^{-}[523] \nu 5 / 2^{-}[523] \nu 11 / 2^{-}[505]\right)$ configuration at 2314 $\mathrm{keV}$ and a $23 / 2^{+}\left(\pi 7 / 2^{-}[523] v 5 / 2^{+}[642] v 11 / 2^{-}[505]\right)$ configuration at $2334 \mathrm{keV}$. The intrinsic $g$ factors extracted from the intensities in Table II give $\left|g_{K}-g_{R}\right| \leqslant 0.037$ compared to values of $\left|g_{K}-g_{R}\right|=0.11(5)$ for the $23 / 2^{-}$

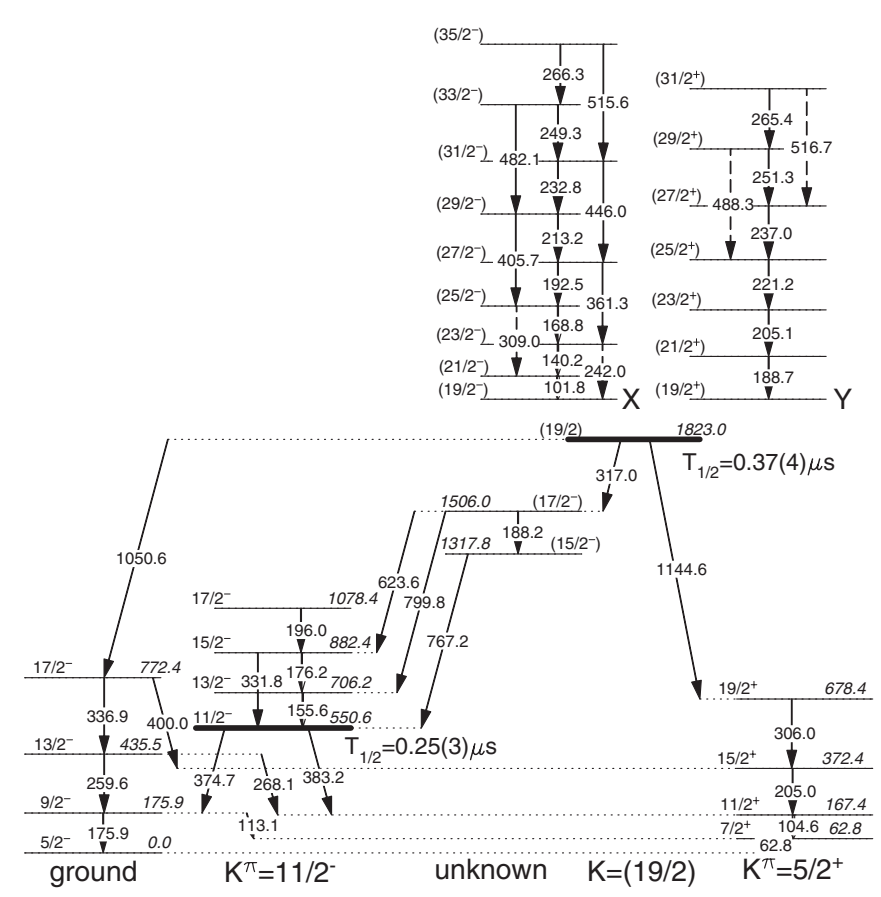

FIG. 16. Partial level scheme for ${ }^{165}$ Er showing the decay of the new isomer $\left(T_{1 / 2}=0.37(4) \mu \mathrm{s}\right)$. Spin and parity assignments for $\mathrm{X}$ and $\mathrm{Y}$ are tentative. 
TABLE V. $\gamma$-ray energy, relative intensity, initial level energy, initial angular momentum, and final angular momentum for the two bands found to be feeding the new isomer in ${ }^{165} \mathrm{Er}$. Top: Band $\mathrm{X}$ (see Fig. 16). Bottom: Band Y (see Fig. 16). Asterisks denote $\Delta I=2$ transitions. The uncertainty in the $\gamma$-ray energies is typically $0.1 \mathrm{keV}$.

\begin{tabular}{lcccc}
\hline \hline$E_{\gamma}(\mathrm{keV})$ & $I_{\gamma}$ & $E_{i}$ & $I_{i}^{\pi}$ & $I_{f}^{\pi}$ \\
\hline 101.8 & $40(3)$ & 1924.8 & $\left(21 / 2^{-}\right)$ & $\left(19 / 2^{-}\right)$ \\
140.2 & $83(8)$ & 2065.0 & $\left(23 / 2^{-}\right)$ & $\left(21 / 2^{-}\right)$ \\
168.8 & $100(7)$ & 2233.8 & $\left(25 / 2^{-}\right)$ & $\left(23 / 2^{-}\right)$ \\
192.5 & $75(6)$ & 2426.3 & $\left(27 / 2^{-}\right)$ & $\left(25 / 2^{-}\right)$ \\
213.2 & $61(7)$ & 2639.5 & $\left(29 / 2^{-}\right)$ & $\left(27 / 2^{-}\right)$ \\
232.8 & $51(5)$ & 2872.3 & $\left(31 / 2^{-}\right)$ & $\left(29 / 2^{-}\right)$ \\
249.3 & $45(6)$ & 3121.6 & $\left(33 / 2^{-}\right)$ & $\left(31 / 2^{-}\right)$ \\
266.3 & $27(6)$ & 3387.9 & $\left(35 / 2^{-}\right)$ & $\left(33 / 2^{-}\right)$ \\
$(242.0)^{*}$ & $19(7)$ & 2065.0 & $\left(23 / 2^{-}\right)$ & $\left(19 / 2^{-}\right)$ \\
$(309.0)^{*}$ & $24(8)$ & 2233.8 & $\left(25 / 2^{-}\right)$ & $\left(21 / 2^{-}\right)$ \\
$361.3^{*}$ & $26(4)$ & 2426.3 & $\left(27 / 2^{-}\right)$ & $\left(23 / 2^{-}\right)$ \\
$405.7^{*}$ & $30(10)$ & 2639.5 & $\left(29 / 2^{-}\right)$ & $\left(25 / 2^{-}\right)$ \\
$446.0^{*}$ & $25(3)$ & 2872.3 & $\left(31 / 2^{-}\right)$ & $\left(27 / 2^{-}\right)$ \\
$482.1^{*}$ & $40(7)$ & 3121.6 & $\left(33 / 2^{-}\right)$ & $\left(29 / 2^{-}\right)$ \\
$515.6^{*}$ & $34(6)$ & 3387.9 & $\left(35 / 2^{-}\right)$ & $\left(31 / 2^{-}\right)$ \\
\hline 188.7 & $95(6)$ & 2011.7 & $\left(21 / 2^{+}\right)$ & $\left(19 / 2^{+}\right)$ \\
205.1 & $66(6)$ & 2216.8 & $\left(23 / 2^{+}\right)$ & $\left(21 / 2^{+}\right)$ \\
221.2 & $70(10)$ & 2438.0 & $\left(25 / 2^{+}\right)$ & $\left(23 / 2^{+}\right)$ \\
237.0 & $46(7)$ & 2675.0 & $\left(27 / 2^{+}\right)$ & $\left(25 / 2^{+}\right)$ \\
251.3 & $40(6)$ & 2926.3 & $\left(29 / 2^{+}\right)$ & $\left(27 / 2^{+}\right)$ \\
265.4 & $40(6)$ & 3191.7 & $\left(31 / 2^{+}\right)$ & $\left(29 / 2^{+}\right)$ \\
$(488.3)^{*}$ & $25(6)$ & 2926.3 & $\left(29 / 2^{+}\right)$ & $\left(25 / 2^{+}\right)$ \\
$(516.7)^{*}$ & $32(6)$ & 3191.7 & $\left(31 / 2^{+}\right)$ & $\left(27 / 2^{+}\right)$ \\
\hline \hline & & & &
\end{tabular}

configuration and $\left|g_{K}-g_{R}\right|=0.02(5)$ for the $23 / 2^{+}$configuration. This $g$-factor evidence favors the $23 / 2^{+}$configuration.

The low-energy $\Delta I=1$ transitions for this band correspond to a high alignment (Fig. 17), suggesting the presence of the $5 / 2^{+}[642]$ neutron and supporting the $23 / 2^{+}$configuration. Thus, we tentatively assign a spin-parity of $23 / 2^{+}$for the $2109-\mathrm{keV}$ isomer.

\section{B. ${ }^{162} \mathrm{Er}$}

For the ${ }^{162} \mathrm{Er}$ isomer presented in Sec. IIIC, the spin 7 assignment for this level, reported by De Boer et al. [23] and Janssens et al. [22] on the basis of angular distributions, was supported by the similarity of the isomer's decay with that of a $7^{-}$isomer in ${ }^{164} \mathrm{Er}$. BCS calculations support this assignment further, predicting the $7^{-}\left(\pi 7 / 2^{-}[523] \pi 7 / 2^{+}[404]\right)$ only $4 \mathrm{keV}$ from the experimental energy of the isomer (see Table VI). In the absence of known two-quasiproton structures in ${ }^{162} \mathrm{Er}$, a proton pairing strength of $G_{p}=21.5 / A \mathrm{MeV}$ was used as this predicts the energy of the $7^{-}$configuration in ${ }^{164} \mathrm{Er}$. Other $I=7$ configurations were predicted at least $300 \mathrm{keV}$ above the experimental energy. No band was detected above the isomer, ruling out $g$ factor and alignment comparisons.

Both $E 1$ transitions from the isomer have a reduced hindrance of $f_{v}=33$ (see Table III). This value may be compared with other $7^{-}$isomers across the Segrè chart,

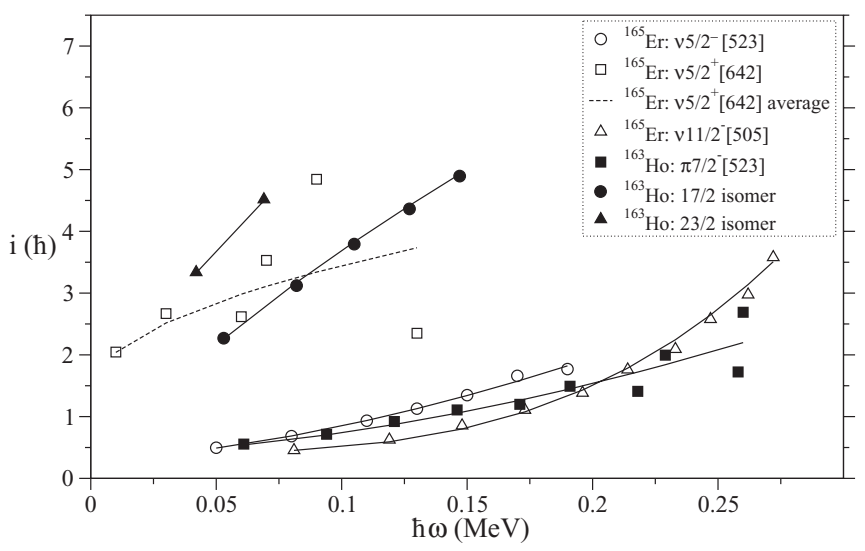

FIG. 17. Angular momentum alignment with the rotation axis for the bands identified in ${ }^{163} \mathrm{Ho}$. One-quasineutron bands from ${ }^{165} \mathrm{Er}$ are compared. The Harris parameters are $\mathcal{J}_{0}=37 \hbar^{2} \mathrm{MeV}^{-1}$ and $\mathcal{J}_{1}=42 \hbar^{4} \mathrm{MeV}^{-3}$ [34].

revealing the effect of $K$ mixing on their decay rates. Figure 18 plots reduced hindrance as a function of the ground-stateband ratio of dynamic $\left[\mathcal{J}^{1}=(2 I-1) \hbar / E_{\gamma}(I)\right]$ and kinematic $\left(\mathcal{J}^{2}=4 \hbar^{2} /\left[E_{\gamma}(I+2)-E_{\gamma}(I)\right]\right)$ moments of inertia. This ratio gives a measure of rotational alignment in the groundstate band, indicating the degree of $K$ mixing in the states fed by the isomer $[8,9]$.

All of the isomers in Fig. 18 possess a remarkably similar half-life ( $5 \mathrm{~ns} \leqslant T_{1 / 2} \leqslant 100 \mathrm{~ns}$ ), and this is reflected in their reduced hindrances, which are close to constant (the low value for ${ }^{128} \mathrm{Ba}$ is discussed later). In stark contrast, and building on Ref. [9], Fig. 19 is the comparative plot for $8^{-}$isomers, showing a significant reduction in reduced hindrance as Coriolis $K$ mixing within the ground-state band increases, before plateauing around the level shown in Fig. 18.

The lack of this relationship in Fig. 18 can be interpreted as due to $K$ mixing within the parent states of the ${ }^{162} \mathrm{Er},{ }^{164} \mathrm{Er}$,

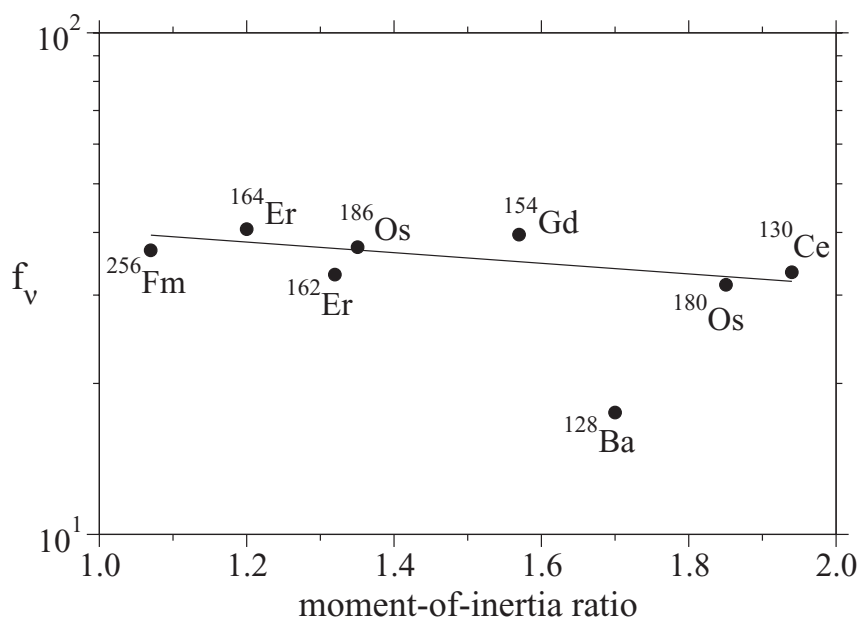

FIG. 18. Reduced hindrance values for $\Delta K=7, E 1$ transitions from two-quasiparticle isomers in even-even nuclei [24,35-40]. The horizontal axis is the ratio of the ground-state-band dynamic and kinematic moments of inertia. The line is to guide the eye. 
TABLE VI. Calculated energies $\left(E_{\mathrm{bcs}}\right)$ for multi-quasiparticle states compared with experimentally determined energies $\left(E_{\text {exp }}\right)$.

\begin{tabular}{|c|c|c|c|c|c|c|c|c|}
\hline \multirow[t]{3}{*}{${ }^{163} \mathrm{Ho}$} & \multirow[t]{3}{*}{1506} & \multirow[t]{3}{*}{0.258} & \multirow[t]{3}{*}{0.007} & \multirow[t]{3}{*}{$19.50 / A$} & \multirow[t]{3}{*}{$21.25 / A$} & $17 / 2^{+}\left(\pi 7 / 2^{-}[523] \nu 5 / 2^{-}[523] \nu 5 / 2^{+}[642]\right)$ & $1504^{\mathrm{c}}$ & $*(\mathrm{~A})$ \\
\hline & & & & & & $17 / 2^{-}\left(\pi 7 / 2^{+}[404] v 5 / 2^{-}[523] v 5 / 2^{+}[642]\right)$ & 1944 & $*(\mathrm{~B})$ \\
\hline & & & & & & $17 / 2^{-}\left(\pi 7 / 2^{-}[523] \pi 7 / 2^{+}[404] \pi 3 / 2^{+}[411]\right)$ & 1781 & $*(\mathrm{C})$ \\
\hline \multirow[t]{2}{*}{${ }^{163} \mathrm{Ho}$} & \multirow[t]{2}{*}{2109} & \multirow[t]{2}{*}{0.258} & \multirow[t]{2}{*}{0.007} & \multirow[t]{2}{*}{$19.50 / A$} & \multirow[t]{2}{*}{$21.25 / A$} & $23 / 2^{-}\left(\pi 7 / 2^{-}[523] v 5 / 2^{-}[523] v 11 / 2^{-}[505]\right)$ & 2314 & * \\
\hline & & & & & & $23 / 2^{+}\left(\pi 7 / 2^{-}[523] v 5 / 2^{+}[642] v 11 / 2^{-}[505]\right)$ & 2334 & $*$ \\
\hline${ }^{162} \mathrm{Er}$ & 2027 & 0.250 & -0.007 & $20.23 / A^{\mathrm{d}}$ & $21.50 / A^{\mathrm{e}}$ & $7^{+}\left(v 3 / 2^{-}[521] v 11 / 2^{-}[505]\right)$ & 2333 & \\
\hline \multirow[t]{4}{*}{${ }^{165} \mathrm{Er}$} & \multirow[t]{4}{*}{1823} & \multirow[t]{4}{*}{0.258} & \multirow[t]{4}{*}{0.013} & \multirow[t]{4}{*}{$19.85 / A^{\mathrm{e}}$} & \multirow[t]{4}{*}{$21.50 / A^{\mathrm{e}}$} & $19 / 2^{+}\left(\nu 5 / 2^{-}[523] \pi 7 / 2^{-}[523] \pi 7 / 2^{+}[404]\right)$ & 1893 & $*$ \\
\hline & & & & & & $19 / 2^{-}\left(\nu 5 / 2^{+}[642] \pi 7 / 2^{-}[523] \pi 7 / 2^{+}[404]\right)$ & 1940 & $*$ \\
\hline & & & & & & $21 / 2^{-}\left(\nu 7 / 2^{+}[633] \pi 7 / 2^{-}[523] \pi 7 / 2^{+}[404]\right)$ & 2359 & \\
\hline & & & & & & $21 / 2^{-}\left(v 11 / 2^{-}[505] v 5 / 2^{-}[523] v 5 / 2^{-}[512]\right)$ & 2377 & \\
\hline
\end{tabular}

$\overline{\mathrm{a}} \epsilon_{2}$ and $\epsilon_{4}$ deformations are from Ref. [30].

${ }^{\mathrm{b}}$ Asterisk denotes that the configuration is discussed in the text.

${ }^{c}$ Fitted to the experimental energy by adjusting $\mathrm{G}_{n}$ and $\mathrm{G}_{p}$.

${ }^{\mathrm{d}}$ Fitted to the energy of a known two-quasiparticle excitation.

${ }^{\mathrm{e}}$ Estimated from neighboring nuclei.

and ${ }^{256} \mathrm{Fm}$ isomer decays. In ${ }^{256} \mathrm{Fm}$ the $K^{\pi}=7^{-}$isomer has a $\pi 7 / 2^{+}[633] \pi 7 / 2^{-}[514]$ configuration [40] resulting in a large Coriolis mixing matrix element from the presence of an $i_{13 / 2}$ proton. This can lead to a low reduced hindrance as seen in Fig. 19(b), which compares $Z=72$ and $N=106$, $K^{\pi}=8^{-}$isomers. As discussed in Ref. [41], for $N=106$ the $v 7 / 2^{-}[514] v 9 / 2^{+}[624]$ configuration of the ${ }^{174} \mathrm{Er}$ and ${ }^{176} \mathrm{Yb}$, $K^{\pi}=8^{-}$isomers, which involves an $i_{13 / 2}$ neutron, results in relatively low $f_{v}$ values. Indeed, in ${ }^{178} \mathrm{Hf}(Z=72)$, mixing of this $2 v$ state with the $\pi 7 / 2^{+}[404] \pi 9 / 2^{-}$[514] configuration, which is assigned to all of the isomers in $Z=72$ nuclei, has resulted in a low $f_{v}$ value [43]. The ${ }^{162} \mathrm{Er}$ and ${ }^{164} \mathrm{Er}$, $K^{\pi}=7^{-}$isomers in Fig. 18 have $\pi 7 / 2^{+}[404] \pi 7 / 2^{-}[523]$

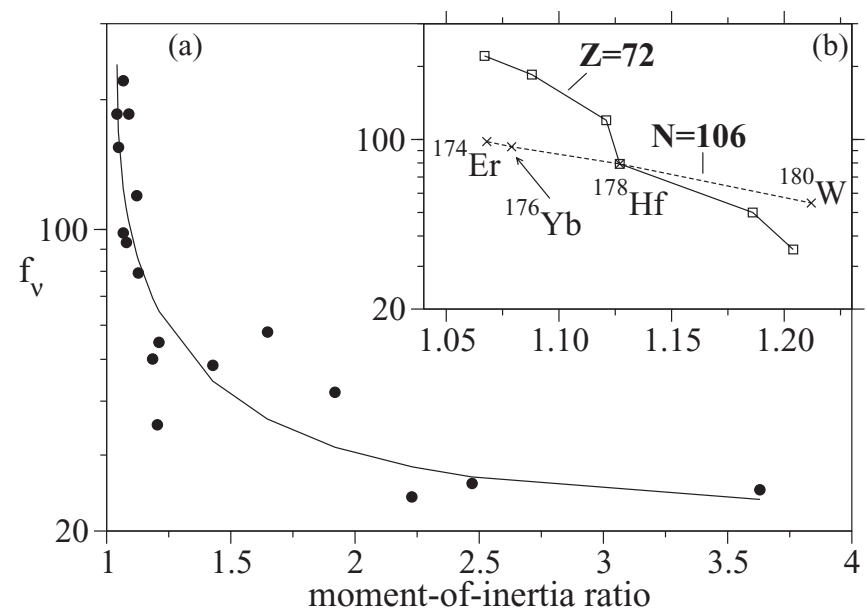

FIG. 19. (a) Reduced hindrance values for $\Delta K=8, E 1$ transitions from two-quasiparticle isomers in even-even nuclei $[9,41,42]$. The horizontal axis is the ratio of the ground-state-band dynamic and kinematic moments of inertia. The line is to guide the eye. (b) Showing [9] only the $Z=72$ and $N=106$ isomers from panel (a). configurations, which like the $Z=72, K^{\pi}=8^{-}$isomers in Fig. 19 do not include an $i_{13 / 2}$ nucleon. However, the lower $\Omega$ value of the $h_{11 / 2}$ orbital in the $K^{\pi}=7^{-}$isomers could contribute to greater Coriolis mixing and a lower reduced hindrance.

Alternatively, the large discrepancy between $K^{\pi}=7^{-}$and $K^{\pi}=8^{-}$isomers at low moment-of-inertia ratios may be attributable to the mixing of a $K^{\pi}=0^{-}$octupole band with the $K^{\pi}=7^{-}$isomers [44]. The low- $K$ admixture of this band will not be seen in $K^{\pi}=8^{-}$isomers due to the symmetry properties of $K^{\pi}=0^{-}$octupole bands, which forbid even-spin states. Octupole mixing with a rotational band built on an isomer has been observed, for example, in ${ }^{170} \mathrm{Yb}$ [45], where a significant reduction in the hindrance of transitions to the ground-state band from the odd-spin states was observed, compared to the transitions from the even-spin states. For $K^{\pi}=7^{-}$isomers, mixing of this variety could reduce the dependence of the reduced hindrance on ground-state-band Coriolis $K$ mixing.

A more quantitative approach to the description of the $K^{\pi}=8^{-}$isomers was taken by Dracoulis et al. [41] by evaluating the Coriolis $K$ mixing in the isomer configuration. It would be useful to extend that approach to the $K^{\pi}=7^{-}$ isomers.

TABLE VII. Intrinsic $g$ factors for the newly identified band $\mathrm{X}$ in ${ }^{165} \mathrm{Er}$. Calculations assume $K=19 / 2$, and a quadrupole moment of $Q_{0}=6.6 \mathrm{~b}$ [30].

\begin{tabular}{ll}
\hline \hline$I_{i}, K$ & $\left|g_{K}-g_{R}\right|$ \\
\hline $27 / 2,19 / 2$ & $0.31_{-3}^{+5}$ \\
$29 / 2,19 / 2$ & $0.33_{-7}^{+10}$ \\
$31 / 2,19 / 2$ & $0.39_{-4}^{+5}$ \\
$33 / 2,19 / 2$ & $0.33_{-5}^{+6}$ \\
$35 / 2,19 / 2$ & $0.31_{-7}^{+7}$ \\
\hline \hline
\end{tabular}




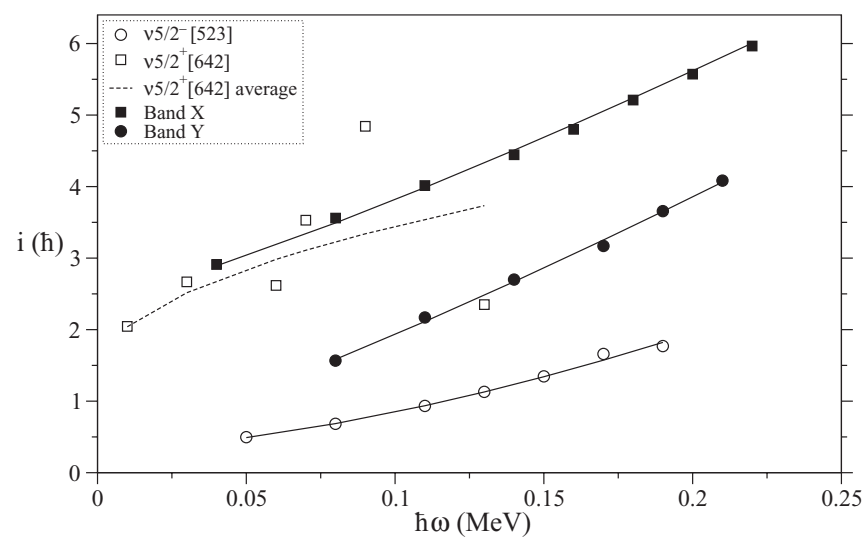

FIG. 20. Net angular momentum alignment for the two new bands, the $v 5 / 2^{+}[642]$ band, and the $\nu 5 / 2^{-}[523]$ band in ${ }^{165} \mathrm{Er}$. The Harris parameters are $\mathcal{J}_{0}=37 \hbar^{2} \mathrm{MeV}^{-1}$ and $\mathcal{J}_{1}=42 \hbar^{4} \mathrm{MeV}^{-3}$ [34].

There is one outlier in Fig. 18, the ${ }^{128} \mathrm{Ba}$ isomer, which can be explained in terms of additional $K$ mixing within the isomer state due to a chance near-degeneracy. A $7^{-}$ level which decays via a $T_{1 / 2}=3.6 \mathrm{ps}, E 1$ transition to the ground-state band is observed $17 \mathrm{keV}$ above the energy of the 6.1-ns isomer [35]. A two-band mixing analysis reveals that a mixing matrix element of $0.43 \mathrm{keV}$ leads to a $0.06 \%$ admixture of the fast decaying state into the isomer, sufficient to explain the low hindrance of its decay [3].

\section{C. ${ }^{165} \mathrm{Er}$}

The daughter-level spins following two transitions from the newly discovered isomer in ${ }^{165} \mathrm{Er}$ (see Fig. 16) indicated a parent-level spin of between $17 / 2$ and $21 / 2$. BCS calculations predict a $19 / 2^{+}\left(\nu 5 / 2^{-}[523] \pi 7 / 2^{-}[523] \pi 7 / 2^{+}[404]\right)$ configuration $70 \mathrm{keV}$ above the experimental energy of $1823 \mathrm{keV}$ and a $19 / 2^{-}\left(\nu 5 / 2^{+}[642] \pi 7 / 2^{-}[523] \pi 7 / 2^{+}\right.$[404]) configuration $117 \mathrm{keV}$ above the experimental energy (see Table VI). The likelihood of these two configurations being the bands labeled $\mathrm{X}$ and $\mathrm{Y}$ in Fig. 16 is enhanced by the remarkable similarity with the level structure observed in ${ }^{163} \mathrm{Er}$ [46].

Intrinsic $g$ factors were extracted for band $\mathrm{X}$, which has confirmed $\Delta I=2$ transitions (see top half of Table $\mathrm{V}$ for intensities). These are summarized in Table VII and indicate $\left|g_{K}-g_{R}\right|=0.33(2)$. Using the values detailed in Sec. IV A, the theoretical $g$ factor for the $19 / 2^{+}$configuration was calculated to be $\left|g_{K}-g_{R}\right|=0.56(5)$, and for the $19 / 2^{-}$configuration it was calculated to be $\left|g_{K}-g_{R}\right|=0.46(5)$. The $g$ factors marginally favor the $19 / 2^{-}$configuration for band X.
As the $19 / 2^{-}$and $19 / 2^{+}$configurations both contain the $7^{-}\left(\pi 7 / 2^{-}[523] \pi 7 / 2^{+}[404]\right)$ two-quasiproton excitation coupled with either the $v 5 / 2^{+}[642]$ neutron or the $v 5 / 2^{-}[523]$ neutron, there should be a considerable difference in the alignment of the two configurations, reflected by the difference in alignment for the one-quasineutron $v 5 / 2^{+}[642]$ and $\nu 5 / 2^{-}$[523] bands in ${ }^{165}$ Er. Figure 20 plots the alignment of these structures, confirming that band $\mathrm{X}$ is more aligned than band $\mathrm{Y}$ by a difference approximately equal to the difference in aligment of the $v 5 / 2^{+}[642]$ and $\nu 5 / 2^{-}$[523] bands. This is strong evidence that band $\mathrm{X}$ contains the $\nu 5 / 2^{+}[642]$ neutron from the $19 / 2^{-}$configuration, agreeing with the $g$-factor measurement. We therefore favor a bandhead spin-parity of $19 / 2^{-}$for band $\mathrm{X}$ and $19 / 2^{+}$for $\mathrm{Y}$.

It is not possible to assign the $19 / 2^{+}$or $19 / 2^{-}$bands to the isomer. We can only state that both bands decay through the isomer, because both are in early coincidence with respect to the delayed isomeric transitions. It is highly likely that one of these bands is the isomer band, while the other decays into the isomer bandhead through a low-energy $E 1$ transition $(<60 \mathrm{keV})$. This transition may be obscured by strong $\mathrm{x}$ rays between 40 and $60 \mathrm{keV}$.

\section{SUMMARY}

Four isomers have been discovered in $N=94-97$ dysprosium, holmium, and erbium isotopes. Half-life measurements were obtained and structural assignments were proposed on the basis of decay-branch transition rates, multi-quasiparticle calculations, and the intrinsic $g$ factor and alignment of their associated rotational bands.

An isomer in ${ }^{162} \mathrm{Er}$, which decays by $E 1$ transitions to the ground-state band with a reduced hindrance of $f_{v}=33$, is compared with other $K^{\pi}=7^{-}$isomers with similar decay characteristics on a plot of $f_{v}$ versus the dynamic to kinematic moment-of-inertia ratio. The near constant $f_{v}$ values over the full moment-of-inertia ratio range, a feature not seen for $K^{\pi}=$ $8^{-}$isomers, suggests the dominant $K$ mixing in these isomers occurs in the isomer itself rather than in the daughter state. This mixing is interpreted as being caused by a combination of Coriolis effects due to the occupation of high- $j$, lower- $\Omega$ orbitals in $K^{\pi}=7^{-}$isomers and band mixing from the $K^{\pi}=$ $0^{-}$octupole band.

\section{ACKNOWLEDGMENTS}

This work has been supported by the UK STFC and the AWE plc.
[1] P. M. Walker and G. D. Dracoulis, Nature (London) 399, 35 (1999).

[2] P. M. Walker and G. D. Dracoulis, Hyperfine Interact. 135, 83 (2001).

[3] G. D. Dracoulis, F. G. Kondev, G. J. Lane, A. P. Byrne, T. R. McGoram, T. Kibédi, I. Ahmad, M. P. Carpenter, R. V. F. Janssens, T. Lauritsen, C. J. Lister, D. Seweryniak,
P. Chowdhury, and S. K. Tandel, Phys. Rev. Lett. 97, 122501 (2006).

[4] F. S. Stephens, Rev. Mod. Phys. 47, 1 (1975).

[5] P. M. Walker, D. M. Cullen, C. S. Purry, D. E. Appelbe, A. P. Byrne, G. D. Dracoulis, T. Kibédi, F. G. Kondev, I. Y. Lee, A. O. Macchiavelli, A. T. Reed, P. H. Regan, and F. Xu, Phys. Lett. B 408, 42 (1997). 
[6] G. J. Lane, G. D. Dracoulis, F. G. Kondev, R. O. Hughes, H. Watanabe, A. P. Byrne, M. P. Carpenter, C. J. Chiara, P. Chowdhury, R. V. F. Janssens, T. Lauritsen, C. J. Lister, E. A. McCutchan, D. Seweryniak, I. Stefanescu, and S. Zhu, Phys. Rev. C 82, 051304(R) (2010).

[7] T. P. D. Swan, P. M. Walker, Zs. Podolyák, M. W. Reed, G. D. Dracoulis, G. J. Lane, T. Kibdi, and M. L. Smith, Phys. Rev. C 83, 034322 (2011).

[8] P. M. Walker, G. D. Dracoulis, A. P. Byrne, T. Kibedi, and A. E. Stuchbery, Phys. Rev. C 49, 1718 (1994).

[9] P. M. Walker, Nucl. Phys. A 834, 22c (2010).

[10] G. D. Dracoulis, Nucl. Phys. News 9, 1 (1999).

[11] T. P. D. Swan, P. M. Walker, Zs. Podolyák, M. W. Reed, G. D. Dracoulis, G. J. Lane, T. Kibdi, and M. L. Smith (to be published).

[12] G. D. Dracoulis and A. P. Byrne, ANU-P/1052(1995)115, 1995 (unpublished).

[13] A. Jungclaus, B. Binder, A. Dietrich, T. Hartlein, H. Bauer, Ch. Gund, D. Pansegrau, D. Schwalm, D. Bazzacco, E. Farnea, S. Lunardi, C. Rossi-Alvarez, C. Ur, G. de Angelis, A. Gadea, D. R. Napoli, X. R. Zhou, and Y. Sun, Phys. Rev. C 67, 034302 (2003).

[14] V. A. Bondarenko et al., in Proceedings of the 33rd Annual Conference on Nuclear Spectroscopic Structures in Atomic Nuclei, Moscow, 1983.

[15] T. Kibédi, T. W. Burrows, M. B. Trzhaskovskaya, P. M. Davidson, and C. W. Nestor Jr., Nucl. Instr. Meth. A 589, 202 (2008).

[16] P. O. Tjom and B. Elbek, Mat. Fys. Medd. Dan. Vidensk. Selsk. 37, 7 (1969).

[17] W. Andrejtscheff, P. Manfrass, H. Prade, K. D. Schilling, G. Winter, H. Fuia, R. Ion-Mihai, A. B. Khalikulov, V. A. Morozov, N. Z. Marupov, and T. M. Muminov, Nucl. Phys. A 220, 438 (1974).

[18] D. Hojman, M. A. Cardona, D. Bazzacco, N. Blasi, J. Davidson, M. Davidson, M. E. Debray, A. J. Kreiner, S. M. Lenzi, G. Lo Bianco, D. R. Napoli, and C. Rossi Alvarez, Eur. Phys. J. A 21, 383 (2004).

[19] K. E. G. Lobner, Phys. Lett. B 26, 369 (1968).

[20] R. B. Firestone and V. S. Shirley (editors), Table of Isotopes, 8th ed. (Wiley, New York, 1996).

[21] J. F. Smith, S. J. Dorning, B. J. Varley, W. R. Phillips, Ch-. Vieu, J. S. Dionisio, C. Schuck, and M. Pautrat, Phys. Rev. C 62, 034312 (2000).

[22] R. Janssens, Y. El Masri, J. M. Ferté, C. Michel, J. Steyaert, and J. Vervier, Nucl. Phys. A 283, 493 (1977).

[23] F. W. N. De Boer, P. F. A. Goudsmit, B. J. Meijer, P. Koldewijn, J. Konijn, and R. Beetz, Nucl. Phys. A 236, 349 (1974).

[24] F. W. N. De Boer, P. F. A. Goudsmit, P. Koldewijn, and B. J. Meijer, Nucl. Phys. A 169, 577 (1971).

[25] A. K. Jain, R. K. Sheline, P. C. Sood, and Kiran Jain, Rev. Mod. Phys. 62, 2 (1990).

[26] S. T. Wang, X. H. Zhou, Y. H. Zhang, Z. Y. Sun, Y. Zheng, M. L. Liu, L. Chen, N. T. Zhang, J. Hu, F. Ma, W. Hua, S. Guo, Y. H. Qiang, L. Ma, Y. D. Fang, G. S. Li, H. B. Zhou, B. Ding, H. X.
Wang, X. G. Lei, Y. X. Guo, Y. X. Liu, Y. Shi, F. R. Xu, L. H. Zhu, and X. G. Wu, Phys. Rev. C 84, 037303 (2011).

[27] K. Jain, O. Burglin, G. D. Dracoulis, B. Fabricius, N. Rowley, and P. M. Walker, Nucl. Phys. A 591, 61 (1995).

[28] H. J. Jensen, R. A. Bark, P. O. Tjom, G. B. Hagemann, I. G. Bearden, H. Carlsson, S. Leoni, T. Lonnroth, W. Reviol, L. L. Riedinger, H. Schnack-Petersen, T. Shizuma, X. Z. Wang, and J. Wrzesinski, Nucl. Phys. A 695, 3 (2001).

[29] A. Bohr and B. R. Mottelson, Nuclear Structure (Benjamin, New York, 1975), Vol. 2.

[30] P. Moller, J. R. Nix, W. D. Myers, and W. J. Swiatecki, At. Data Nucl. Data Tables 59, 185 (1995).

[31] P. M. Walker, G. D. Dracoulis, A. P. Byrne, B. Fabricius, T. Kibédi, A. E. Stuchbery, and N. Rowley, Nucl. Phys. A 568, 397 (1994).

[32] T. P. D. Swan, Ph.D. thesis, University of Surrey, 2011.

[33] S. M. Mullins, G. D Dracoulis, A. P Byrne, T. R McGoram, S. Bayer, W. A Seale, and F. G Kondev, Phys. Lett. B 393, 279 (1997).

[34] S. Frauendorf, Phys. Scr. 24, 349 (1981).

[35] O. Vogel, R. S. Chakrawarthy, A. Dewald, P. Petkov, K. Jessen, J. Gableske, P. von Brentano, D. Bazzacco, A. Gizon, J. Gizon, S. Lunardi, D. R. Napoli, P. Pavan, C. Rossi Alvarez, and I. Wiedenhver, Eur. Phys. J. A 4, 323 (1999).

[36] D. M. Todd, R. Aryaeinejad, D. J. G. Love, A. H. Nelson, P. J. Nolan, P. J. Smith, and P. J. Twin, J. Phys. G 10, 1407 (1984).

[37] J. F. Sharpey-Schafer, T. E. Madiba, S. P. Bvumbi, E. A. Lawrie, J. J. Lawrie, A. Minkova, S. M. Mullins, P. Papka, D. G. Roux, and J. Timár, Eur. Phys. J. A 47, 5 (2011).

[38] R. M. Lieder, Ts. Venkova, S. Utzelmann, W. Gast, H. Schnare, K. Spohr, P. Hoernes, A. Georgiev, D. Bazzacco, R. Menegazzo, C. Rossi-Alvarez, G. de Angelis, R. Kaczarowski, T. RzaccaUrban, T. Morek, G. V. Marti, K. H. Maier, and S. Frauendorf, Nucl. Phys. A 645, 465 (1999).

[39] C. Wheldon, P. M. Walker, P. H. Regan, T. Saitoh, N. Hashimoto, G. Sletten, and F. R. Xu, Nucl. Phys. A 652, 103 (1999).

[40] F. R. Xu, E. G. Zhao, R. Wyss, and P. M. Walker, Phys. Rev. Lett. 92, 252501 (2004).

[41] G. D. Dracoulis, G. J. Lane, F. G. Kondev, H. Watanabe, D. Seweryniak, S. Zhu, M. P. Carpenter, C. J. Chiara, R. V. F. Janssens, T. Lauritsen, C. J. Lister, E. A. Mc-Cutchan, and I. Stefanescu, Phys. Rev. C 79, 061303(R) (2009).

[42] International Network of Nuclear Structure and Decay Data Evaluators, Evaluated Nuclear Structure and Decay Data File (ENSDF) [http://www-nds.iaea.org/nsdd].

[43] T. L. Khoo and G. Lovhoiden, Phys. Lett. B 67, 271 (1977).

[44] P. M. Walker, Hyperfine Interact. 143, 143 (2002).

[45] P. M. Walker, S. R. Faber, W. H. Bentley, R. M. Ronningen, R. B. Firestone, and F. M. Bernthal, Phys. Lett. B 86, 1 (1979).

[46] G. B. Hagemann, H. Ryde, P. Bosetti, A. Brockstedt, H. Carlsson, L. P. Ekstrom, A. Nordlund, R. A. Bark, B. Herskind, S. Leoni, A. Bracco, F. Camera, S. Frattini, M. Mattiuzzi, B. Million, C. Rossi-Alvarez, G. de Angelis, D. Bazzacco, S. Lunardi, and M. De Poli, Nucl. Phys. A 618, 199 (1997). 